\title{
OBSERVATIONS OF INTENSITY FLUCTUATIONS ATTRIBUTED TO GRANULATION AND FACULAE ON SUN-LIKE STARS FROM THE KEPLER MISSION
}

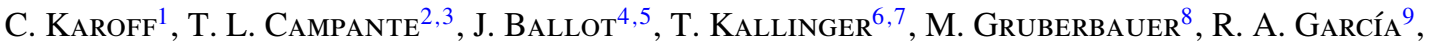 \\ D. A. Caldwell ${ }^{10}$, J. L. Christiansen ${ }^{10}$, and K. Kinemuchi ${ }^{11}$ \\ ${ }^{1}$ Stellar Astrophysics Centre, Department of Physics and Astronomy, Aarhus University, Ny Munkegade 120, DK-8000 Aarhus C, Denmark; karoff@phys.au.dk \\ ${ }^{2}$ Centro de Astrofísica and Faculdade de Ciências, Universidade do Porto, Rua das Estrelas, 4150-762 Porto, Portugal \\ ${ }^{3}$ School of Physics and Astronomy, University of Birmingham, Edgbaston, Birmingham, B15 2TT, UK \\ ${ }^{4}$ CNRS, Institut de Recherche en Astrophysique et Planétologie, 14 avenue Édouard Belin, F-31400, Toulouse, France \\ ${ }^{5}$ Université de Toulouse, UPS-OMP, IRAP, Toulouse, France \\ ${ }^{6}$ Instituut voor Sterrenkunde, K. U. Leuven, Celestijnenlaan 200D, B-3001 Leuven, Belgium \\ ${ }^{7}$ Institute for Astronomy, University of Vienna, Türkenschanzstrasse 17, A-1180 Vienna, Austria \\ ${ }^{8}$ Institute for Computational Astrophysics, Department of Astronomy and Physics, Saint Mary's University, B3H 3C3 Halifax, Canada \\ ${ }^{9}$ Laboratoire AIM, CEA/DSM-CNRS-Universit Paris Diderot, IRFU/SAp, Centre de Saclay, F-91191 Gif-sur-Yvette Cedex, France \\ ${ }^{10}$ SETI Institute/NASA Ames Research Center, Moffett Field, CA 94035, USA \\ ${ }^{11}$ Bay Area Environmental Research Inst./NASA Ames Research Center, Moffett Field, CA 94035, USA \\ Received 2012 July 4; accepted 2013 February 21; published 2013 March 22
}

\begin{abstract}
Sun-like stars show intensity fluctuations on a number of timescales due to various physical phenomena on their surfaces. These phenomena can convincingly be studied in the frequency spectra of these stars-while the strongest signatures usually originate from spots, granulation, and $p$-mode oscillations, it has also been suggested that the frequency spectrum of the Sun contains a signature of faculae. We have analyzed three stars observed for 13 months in short cadence (58.84 s sampling) by the Kepler mission. The frequency spectra of all three stars, as for the Sun, contain signatures that we can attribute to granulation, faculae, and $p$-mode oscillations. The temporal variability of the signatures attributed to granulation, faculae, and $p$-mode oscillations was analyzed and the analysis indicates a periodic variability in the granulation and faculae signatures - comparable to what is seen in the Sun.
\end{abstract}

Key words: stars: activity - stars: individual (KIC 6603624, KIC 6933899, KIC 11244118) - stars: oscillations stars: solar-type

\section{INTRODUCTION}

Low-mass main-sequence stars (Sun-like stars) have an outer convection zone, which means that they show granulation on their surface. The outer convection zone is responsible for exciting acoustic oscillations inside these stars, which, at least in the stars with masses close to the Sun, have observable amplitudes at the surfaces of these stars (Houdek et al. 1999). When observing the disk-integrated intensity of these stars as a function of time, the resulting light curves will thus include the signatures of both granulation and oscillations. These signatures are usually hard to identify directly in the light curves as granulation, which has the largest amplitude, is a strongly noncoherent phenomenon. The same applies to oscillations in Sunlike stars as their oscillations are damped, which means that they are also not coherent on long timescales (Goldreich et al. 1994). An exception from this are evolved stars, which can have clear signatures of both granulation and oscillations in their light curves (Schwarzschild 1975).

Granulation and oscillations are therefore usually studied in Sun-like stars by analyzing the frequency spectrum of their light curves. These frequency spectra show a characteristic decline in power with increasing frequency caused by granulation. The location in frequency of this characteristic decline gives the characteristic timescale of granulation-equivalent to the life time or the turn-over time of the granules, which has been measured in the Sun (Harvey 1985), Sun-like stars (Michel et al. 2008), and evolved red giant stars (Mathur et al. 2011).

The characteristic frequency of the $p$-mode oscillations in Sun-like (and evolved) stars (i.e., the frequency of maximum power) is proportional to the atmospheric acoustic cutoff frequency which is given as the sound speed divided by twice the pressure scale height near the surface-assuming the atmosphere is isothermal (Belkacem et al. 2011). Traditionally, it has been assumed that the characteristic timescale of granulation would also scale with the atmospheric acoustic cutoff frequency (Brown et al. 1991; Kjeldsen \& Bedding 1995, 2011). This means that there would be a linear relation between the atmospheric acoustic cutoff frequency and the difference between the characteristic timescale of granulation and the $p$-mode oscillations.

It has been known for some time that the frequency spectrum of the Sun shows another feature in the region located between the characteristic decline in power caused by granulation and the bump caused by the $p$-mode oscillations (Harvey et al. 1993). Lately, it has been proposed that this feature is likely caused by bright faculae on the surface of the Sun (Karoff 2012).

At low frequencies, peaks can be seen caused by the rotational modulation of long-lived sunspots (Lanza et al. 2004). At frequencies below $1 \mu \mathrm{Hz}$ a linear decline in power with increasing frequency can be seen, which is caused by the weak coherency of activity-related phenomena such as sunspots (Harvey et al. 1993). At frequencies higher than the atmospheric acoustic cutoff frequency evidence is seen for the so called high-frequency peaks, which could be traveling waves or chromospheric oscillations (García et al. 1998; Jiménez et al. 2005; Karoff 2007, 2009; Karoff \& Kjeldsen 2008).

At least two different interpretations of the physical processes responsible for the different features in the solar frequency spectrum can be found in the literature. In the first interpretation, which has been used by Andersen et al. (1998), Anklin et al. (1998), Aigrain et al. (2004), and Michel et al. (2009), the different features are explained by rotation, supergranulation, mesogranulation, granulation, and oscillations (see Table 1). 
Table 1

Interpretation of the Physical Processes Responsible for the Different Features in the Solar Frequency Spectrum used by Andersen et al. (1998), Anklin et al. (1998), Aigrain et al. (2004), and Michel et al. (2009)

\begin{tabular}{ll}
\hline \hline $0-1 \mu \mathrm{Hz}$ & Rotation \\
$10-100 \mu \mathrm{Hz}$ & Supergranulation \\
$80-1000 \mu \mathrm{Hz}$ & Mesogranulation \\
$800-3000 \mu \mathrm{Hz}$ & Granulation \\
$2000-4000 \mu \mathrm{Hz}$ & Oscillations \\
\hline
\end{tabular}

Table 2

Interpretation of the Physical Processes Responsible for the Different Features in the Solar Frequency Spectrum used by Harvey et al. (1993), Vázquez Ramió et al. (2002), and Karoff (2012)

\begin{tabular}{ll}
\hline \hline $0-1 \mu \mathrm{Hz}$ & Rotation \\
$100-1000 \mu \mathrm{Hz}$ & Granulation \\
$1000-3000 \mu \mathrm{Hz}$ & Faculae \\
$2000-4000 \mu \mathrm{Hz}$ & Oscillations \\
\hline
\end{tabular}

In the second interpretation, which has been used by Harvey et al. (1993), Vázquez Ramió et al. (2002), and Karoff (2012), they are explained by rotation, granulation, bright points (or faculae), and oscillations (see Table 2). Though this is a rough separation of all these studies into only two different interpretations, it exemplifies the general difference between these studies - that the studies using the first interpretation ascribe the feature just below $1000 \mu \mathrm{Hz}$ to mesogranulation and the feature just above to granulation, whereas the studies using the second interpretation ascribe the feature just below $1000 \mu \mathrm{Hz}$ to granulation and the feature just above to bright points or faculae.

Following Roudier et al. (1998), the three different convective scales can be identified as supergranulation characterized by a mean size of $20-50 \mathrm{Mm}$, mesogranulation by a mean size of 5-10 Mm, and granulation by a mean size of around $1000 \mathrm{~km}$. The coherence timescale of granulation is between 2 and 6 minutes (Del Moro 2004 and references herein). This agrees with the interpretation by Harvey et al. (1993), Vázquez Ramió et al. (2002), and Karoff (2012) that the signature just below $1000 \mu \mathrm{Hz}$ is due to granulation. Mesogranulation and supergranulation would have to have significant longer coherence timescales and thus manifest themselves at frequencies significantly below $1000 \mu \mathrm{Hz}$. No such manifestations seem to be visible in the observed frequency spectrum of the Sun (Karoff 2012).

That the manifestation of mesogranulation and supergranulation is not observable in the frequency spectrum of the Sun does not mean that mesogranulation and supergranulation does not contribute to the background in the frequency spectrum. The contribution is just not large enough to be observable. The same is true for the second population of granules with coherence timescales of around 1 minute identified by Del Moro (2004). It has been shown by Karoff (2012) that this second population of granules, which would be both more numerous and with a lower brightness contrasts, would not be observable in the frequency spectrum, as the amplitude of the signature in the frequency spectrum scales with the brightness contrast over the square root of the number of granules. We thus follow the second interpretation in this paper.

Here we analyze observations from the Kepler spacecraft of the three Sun-like stars: KIC 6603624, KIC 6933899, and KIC 11244118. KIC 6603624 was one of the three stars presented in the first results on solar-like stars based on Kepler observations
Table 3

Stellar Parameters from Asteroseismology

\begin{tabular}{lccc}
\hline \hline KIC & 6603624 & 6933899 & 11244118 \\
\hline$R\left(R_{\odot}\right)$ & $1.15 \pm 0.01$ & $1.58 \pm 0.01$ & $1.55 \pm 0.01$ \\
$M\left(M_{\odot}\right)$ & $1.01 \pm 0.01$ & $1.10 \pm 0.01$ & $1.01 \pm 0.01$ \\
$T_{\text {eff }}(\mathrm{K})$ & 5416 & 5616 & 5507 \\
$K_{\mathrm{p}}(\mathrm{mag})$ & 9.1 & 9.6 & 9.7 \\
Age $(\mathrm{Gyr})$ & $8.51 \pm 0.23$ & $6.28 \pm 0.15$ & $8.93 \pm 0.04$ \\
\hline
\end{tabular}

Note. From Mathur et al. 2012; Verner et al. 2011.

Table 4

Observing Times

\begin{tabular}{lllll}
\hline \hline Quarter & \multicolumn{1}{c}{ Start Data } & & & \\
\hline 1 & 2009 May 13-Jun 17 & $\mathrm{a}$ & & \\
2.1 & 2009 Jun 20-Jul 19 & & $\mathrm{b}$ & $\mathrm{c}$ \\
5 & 2010 Mar 20-Jun 24 & $\mathrm{a}$ & $\mathrm{b}$ & $\mathrm{c}$ \\
6 & 2010 Jun 24-Sep 23 & $\mathrm{a}$ & $\mathrm{b}$ & $\mathrm{c}$ \\
7 & 2010 Sep 23-2011 Jan 6 & $\mathrm{a}$ & $\mathrm{b}$ & $\mathrm{c}$ \\
8 & 2011 Jan 6-Mar 20 & $\mathrm{a}$ & $\mathrm{b}$ & $\mathrm{c}$ \\
\hline
\end{tabular}

Notes. "a” means: KIC 6603624, "b": KIC 6933899, and "c": KIC 11244118.

(Chaplin et al. 2010) and in that work it was already noted that the frequency spectrum of this star shows a number of different components. One component possible originating from bright faculae (as seen in the Sun). The eigenmode frequencies of all three stars were analyzed in detail by Mathur et al. (2012) and the resulting stellar parameters are shown in Table 3. Note that the uncertainties in Table 3 are formal uncertainties only, which do not include estimates of systematic contributions.

The three stars were selected partly because they were among the 22 stars modeled by Mathur et al. (2012), which provides us with precise stellar parameters from asteroseismology and partly because they all show-frequency spectra with features close to those observed in the Sun. The three stars are not the only Sun-like stars observed by Kepler that show the signature that we attribute to faculae in their spectra.

The outline of this paper is as follows. In Section 2 we describe the 13 months of observations from the Kepler spacecraft that were used in this study and how these observations were preprocessed. A description of the analysis of the frequency spectra is given in Section 3, including how we measure the parameters of the components that we attribute to granulation, faculae, and $p$-mode oscillations. The results are presented in Section 4 and a conclusion is given in Section 5.

\section{OBSERVATIONS}

The three stars were all observed in short cadence $(58.85 \mathrm{~s}$ sampling; Gilliland et al. 2010) by the Kepler mission (Koch et al. 2010). The specific observing times are given in Table 4 . The light curves were prepared as described in Jenkins et al. (2010) and García et al. (2011), which removes most instrumental artifacts. The one artifact that was left in the light curves was a general declining trend. As all light curves are normalized to one, it is thus problematic to connect different quarters of observations. This is partly why we have chosen to calculate individual frequency spectra of all months and then combine these spectra to an average spectrum - and because we do not need high-frequency resolution for studying granulation, faculae, and the envelope of the $p$-mode oscillations. 
Apart from the declining trend, all three stars show rms scatter with an amplitude of around $100 \mathrm{ppm}$ in the time domain and the noise level is relatively constant from month to month. The first month in quarter 2 contains a gap, as does the last month of quarter 7 and the second month of quarter 8. Nothing is done to these gaps in the analysis as the normalization to power density (see below) accounts for the missing observations. This means that the measured parameters are not affected by the gaps.

\section{ANALYSIS}

The analysis of the signatures attributed to granulation, faculae, and $p$-mode oscillations in the frequency spectra consists of three steps. First, the frequency spectra are calculated from the light curves, then second, the resulting observed spectra are compared to a model in order to find the best model, and third, the parameters of the components attributed to granulation, faculae, and $p$-mode oscillation are measured.

The power density spectra were calculated using the leastsquares method (Lomb 1976; Karoff 2008). Each spectrum was normalized by the effective observation length given as the reciprocal of the area under the window function in order to convert the spectra into power density and insure that the spectra obey Parseval's theorem.

We have only analyzed the spectra in the frequency range between $100 \mu \mathrm{Hz}$ and up to the frequency of maximum power (as in Karoff 2012). The reason for the lower limit is that this part of the spectrum is often affected by instrumental and rotational effects, which are not the subject of this study. The reason for the higher limit is discussed in details by Karoff (2012) and is related to the choice of model, as we discuss below.

\subsection{The Model}

Traditionally, the signatures attributed to granulation and faculae have been evaluated using the model suggested by Harvey (1985):

$$
f(\nu)=\frac{4 \sigma^{2} \tau}{1+(2 \pi \nu \tau)^{2}},
$$

where $f(v)$ is the power density at frequency $v, \sigma$ is the amplitude of the signature, and $\tau$ is the characteristic timescale.

As discussed in Karoff (2012), various corrections have been made to this equation in order to be able to model the frequency spectrum of the Sun. Here we adopt the model from Karoff (2012):

$$
\begin{aligned}
f(\nu)= & \frac{\zeta_{\text {gran }} \sigma_{\text {gran }}^{2} \tau_{\text {gran }}}{1+\left(2 \pi \nu \tau_{\text {gran }}\right)^{3.5}}+\frac{\zeta_{\text {fac }} \sigma_{\text {fac }}^{2} \tau_{\text {fac }}}{1+\left(2 \pi \nu \tau_{\text {fac }}\right)^{6.2}} \\
& +H_{\text {osc }} \exp \left[\frac{\left(v-v_{\text {max }}\right)^{2}}{2 w^{2}}\right]+\text { Noise. }
\end{aligned}
$$

We have included the normalization constants $\zeta_{\text {gran }}$ and $\zeta_{\text {fac }}$, which we will discuss in the next section. $H_{\mathrm{osc}}$ is the height of the $p$-mode oscillation envelope, $v_{\max }$ is the frequency of maximum power, and $w$ is the width of the $p$-mode oscillation envelope. A white-noise term has also been added to the model.

For the three stars in this study we adopt the solar value of the exponents in Equation (2)-i.e., $-3.5 \pm 0.3$ for granulation and $-6.2 \pm 0.7$ for faculae (Karoff 2012). The size of the exponents is a measurement of the amount of memory in the physical process responsible for the component. A larger exponent means less memory in the process. As the physical processes responsible for granulation and faculae are expected to be the same on other Sun-like stars, with global parameters close to the solar ones, the two exponents are expected to be close to solar values.

As discussed by Karoff (2012), the model in Equation (1) fails to reproduce the observed solar acoustic background for frequencies higher than the atmospheric acoustic cutoff frequency. The reason for this is that granulation cannot be modeled with turbulent cascades (Nordlund et al. 1997) as it is done in the drift model by Harvey (1985). Turbulence shows a distribution with a slope of around -2 in power, convection, on the other hand, has a lower limit in the time domain on which changes can take place. This means that on small timescales (or at high frequency) convection is not noisy whereas turbulence is. Therefore, in order to model the observed solar acoustic background for frequencies higher than the atmospheric acoustic cutoff frequency, an extra term has to be included in Equation (1) (or (2)) as it was done in Karoff (2008). In order not to rely on assumptions about the behavior of this extra term in other stars, we have chosen not to model the high-frequency part of the spectra and this is why we only analyze the spectra up to the frequency of maximum power.

\subsection{Normalization}

The amplitudes of the components attributed to granulation and faculae in Equation (2) can be understood as the variance these signals have in the time series. This is insured by Parseval's theorem, but in order for Equation (2) to obey Parseval's theorem, Equation (2) needs to be normalized using the normalization constants $\zeta_{\text {gran }}$ and $\zeta_{\text {fac }}$.

Parseval's theorem says that

$$
\sum_{\nu=0}^{\nu=v_{\mathrm{Ny}}} f(\nu) \delta \nu=\sum_{t=t_{0}}^{t=t_{N}} \frac{|o(t)-\bar{o}|^{2}}{N} \delta t=\sigma^{2},
$$

where $v_{\mathrm{Ny}}$ is the Nyquist frequency, $N$ is the number of observations, $o(t)$ is the observed intensity at time $t$, and $\bar{o}$ is the mean value of the observations. $\delta t$ is the time step and $\delta v$ is the frequency resolution $[\delta v=(1 / N \delta t)]$. The first sum should be summed over all natural frequencies, whereas the second sum should be summed over all time steps. As we assume the different contributions to the frequency spectra-granulation, faculae, and the $p$-mode oscillation envelope-to be noncoherent and uncorrelated, Parseval's theorem can also be written as a sum of $k$ different contributions:

$$
\sum_{\nu=0}^{\nu=v_{\mathrm{Ny}}} \sum_{k} f_{k}(\nu) \delta \nu=\sigma^{2}=\sum_{k} \sigma_{k}^{2}
$$

and the different contributions can thus be normalized individually to Parseval's theorem, as they are additive in power:

$$
\sum_{\nu=0}^{\nu=v_{\mathrm{Ny}}} f_{k}(v) \delta \nu=\sigma_{k}^{2}
$$

Following Michel et al. (2009), it is thus possible to calculate the normalization constants $\zeta_{\text {gran }}$ and $\zeta_{\text {fac }}$ from Equation (2):

$$
\sum_{\nu=0}^{\nu=\nu_{\mathrm{Ny}}}\left[\frac{\zeta \sigma_{k}^{2} \tau_{k}}{1+\left(2 \pi \nu \tau_{k}\right)^{\alpha_{k}}}\right] \delta \nu=\sigma_{k}^{2}
$$


where $\alpha_{k}$ are the exponents in Equation (2)-i.e., 3.47 for granulation and 6.20 for faculae. Assuming that $v_{\mathrm{Ny}} \tau \gg 1$ it can be shown that

$$
\zeta_{k}=2 \alpha_{k} \sin \left(\frac{\pi}{\alpha_{k}}\right)
$$

Using this formulation we obtain $\zeta_{\text {gran }}=5.46$ and $\zeta_{\text {fac }}=6.02$.

\subsection{Minimization}

The model described above was matched to the observed frequency spectra using maximum-likelihood estimators as described in Karoff (2012) by calculating the logarithmic likelihood function $\ell$ between $N$ independent measurements $x_{i}$-i.e., power density at a given frequency, and the model $f_{i}$ given by a set of parameters $\lambda$ :

$$
\ell=-\sum_{i=1}^{N} \ln p\left(x_{i}, \lambda\right)
$$

where $p\left(x_{i}, \lambda\right)$ is the probability density function, which is obtained from Appourchaux (2004):

$$
p\left[\mathcal{S}_{i}(x, n), f_{i}(\lambda)\right]=\frac{\mu_{i}^{v_{i}}}{\Gamma\left(v_{i}\right)} \mathcal{S}_{i}(x, n)^{v_{i}-1} e^{-\mu \mathcal{S}_{i}(x, n)},
$$

where $S_{i}(x, n)$ is the observed spectrum binned over $n$ bins, $\Gamma$ is the Gamma function, and $\mu_{i}$ and $v_{i}$ are given as

$$
\mu_{i}=\frac{(n+1) \sum_{k=i-n / 2}^{k=i+n / 2} f_{k}(\lambda)}{\sum_{k=i-n / 2}^{k=i+n / 2} f_{k}^{2}(\lambda)},
$$

and

$$
v_{i}=\frac{\left[\sum_{k=i-n / 2}^{k=i+n / 2} f_{k}(\lambda)\right]^{2}}{\sum_{k=i-n / 2}^{k=i+n / 2} f_{k}^{2}(\lambda)} .
$$

Note that $v_{i}$ is different from the $v$ used for the frequencies in the frequency spectra.

The general idea is here to compare a binned version of the observed spectra $S_{i}(x, n)$ to the model in order to calculate the likelihood-instead of assuming that the differences between the observed frequency spectra and the model are given either by a normal distribution or a $\chi^{2}$ distribution with 2 degrees of freedom. The spectrum should be binned over so many bins that the model becomes a good representation of the observed spectrum. On the other hand $n$ should not be so large that the different features in the frequency spectra cannot be observed. We have thus used $n=100$. If $n$ is close to 1, Equation (9) reduces to the well-known case from the modeling of individual $p$-mode oscillation modes where the differences between the observed frequency spectra and the model are given by a $\chi^{2}$ distribution with 2 degrees of freedom (Gabriel 1994).

The minimization of the likelihood function was done using the simplex method as applied in the AMOEBA function (Press et al. 1992).

\subsection{Uncertainties}

Formal uncertainties can be calculated as the diagonal elements of the inverse of the Hessian matrix, but such uncertainties are only internal uncertainties that says something about the curvature of the minimized likelihood function.

The uncertainties also have to take into account the averaging that is performed in the modeling. First we average individual spectra to an average spectrum and then we bin this (and the individual spectra) over $n$ bins. While the first effect can be accounted for by dividing the uncertainties by the square root of the number of individual spectra (Appourchaux 2003) it is not straightforward how to account for the second effect.

If the differences between an observed frequency spectrum sampled at the natural frequencies and the model were assumed to be given by a $\chi^{2}$ distribution with 2 degrees of freedom and this spectrum was binned over $n$ bins, then we know that the differences between the binned spectrum and the model would be given by a $\chi^{2}$ distribution with $2 n$ degrees of freedom. In that case the uncertainties would have to be adjusted accordingly (Appourchaux et al. 2012). As the probability density function that we have used (Equation (9)) accounts for the binning, it is, on the other hand, not clear if any corrections are needed for the uncertainties.

To avoid inconsistencies in the quoted uncertainties, we have chosen to give the average values from the results of different monthly, individual spectra rather than results of an average spectrum. In this way we can also give the uncertainties as the uncertainties on the mean value. This is, on the other hand, not possible to do on the temporal results, so here the error bars only represent formal uncertainties.

\section{RESULTS}

Using the method described above we have modeled a summed spectrum calculated as the sum of individual spectra for each of the 13 months of observations for all three stars. These summed spectra were used to calculate the significance of the component attributed to faculae using the formulation described above. The average spectra are shown in Figure 1 together with the model with (red line) and without (blue line) the component attributed to faculae. We have also analyzed the individual spectra for each of the 13 months of observations for all three stars and the measured mean values and their temporal variability are shown in Figures 4-7.

\subsection{Significance of the Component Attributed to Faculae}

The significance of the signature of the component attributed to faculae was calculated using the logarithmic likelihood ratio $\Lambda$ :

$$
\ln \Lambda=\ell\left(\lambda_{p+q}\right)-\ell\left(\lambda_{p}\right),
$$

where $p$ is the number of free parameters in the model without the component attributed to faculae (i.e., 6) and $q$ is the number of additional parameters in the model with the component attributed to faculae (i.e., 2), and comparing the value of $-2 \ln \Lambda$ to a $\chi^{2}$ distribution with $q$ degrees of freedom as in Karoff (2012).

A visual inspection of the frequency spectra reveals that the model with the component attributed to faculae matches the observed spectra better than the model without such a component in all three cases. This is also reflected in the returned logarithmic likelihood ratios, which are shown in Table 5. The low ratios mean that the significance of the component attributed 

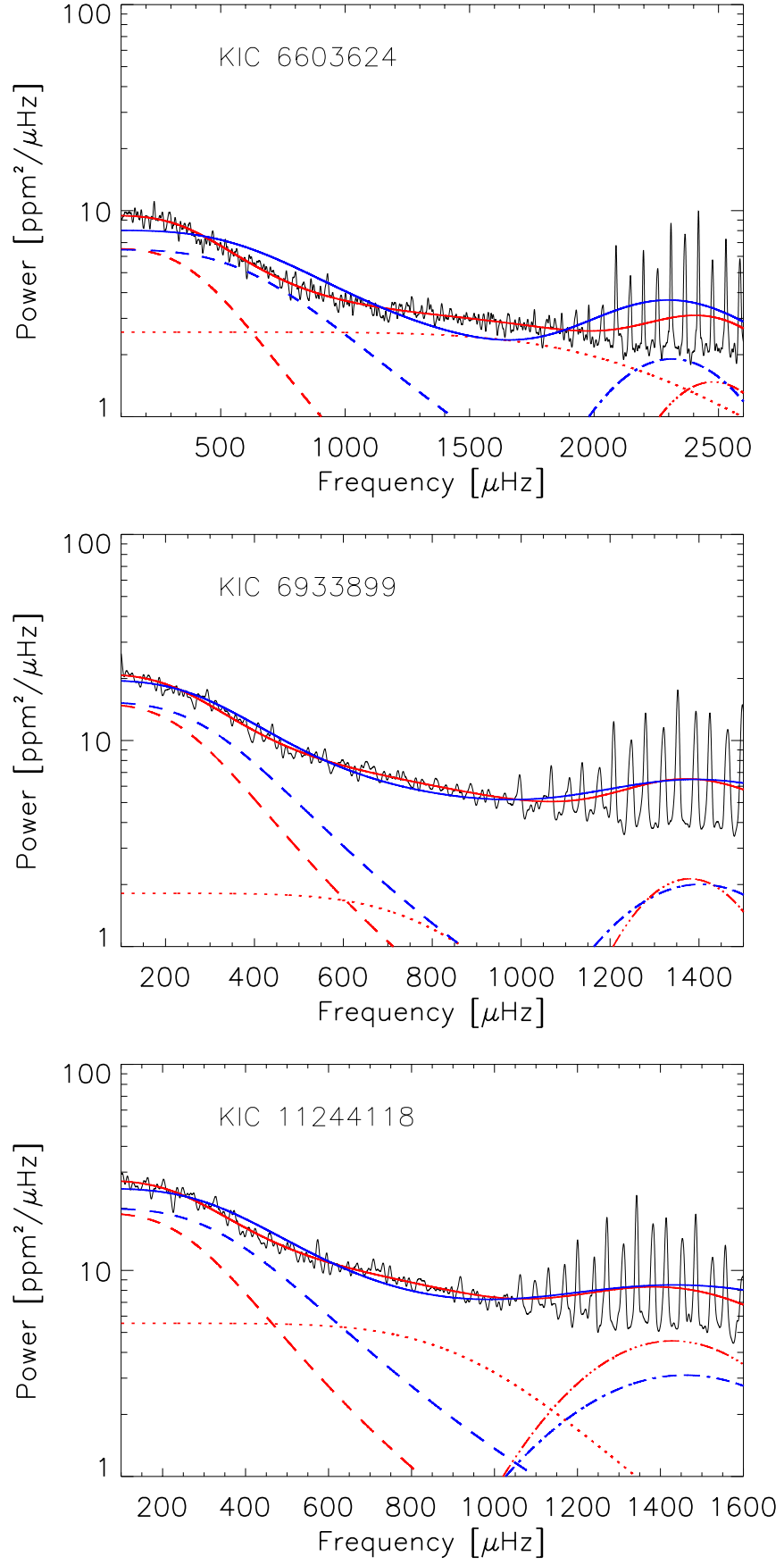

Figure 1. Binned average spectra of KIC 6603624, KIC 6933899, and KIC 11244118 (from top to bottom). The solid red lines are the model with the component attributed to faculae and solid blue lines are the model without the component attributed to faculae. The dashed lines show the component attributed to granulation, whereas the dotted lines show the component attributed to faculae and the dash-dotted lines show the $p$-mode oscillations envelope.

Table 5

Logarithmic Likelihood Ratios $[\ln (\Lambda)]$ —with Fixed and Free Exponents

\begin{tabular}{llcc}
\hline \hline KIC & 6603624 & 6933899 & 11244118 \\
\hline $\ln (\Lambda)_{\text {fixed }}$ & -531.67 & -420.3 & -98.35 \\
$\ln (\Lambda)_{\text {free }}$ & -683.16 & -21.34 & -53.34 \\
\hline
\end{tabular}

to facular is one in all three stars-i.e., the probability of having a logarithmic likelihood ratio smaller than, e.g., -12 returned from a $\chi^{2}$ distribution with 2 degrees of freedom is larger than 0.99999 .

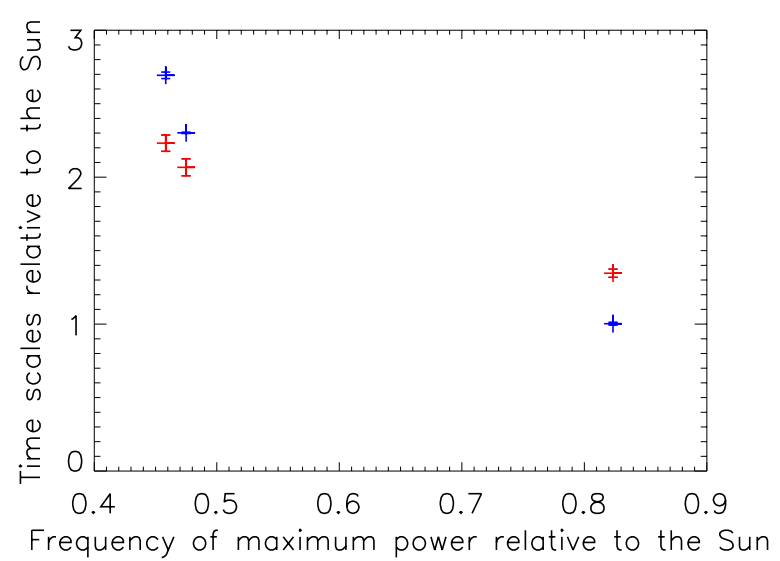

Figure 2. Measured timescales of the components attributed to granulation (red) and faculae (blue) as a function of the frequency of maximum power relative to the Sun. It is generally seen that both timescales decrease with increasing frequency of maximum power.

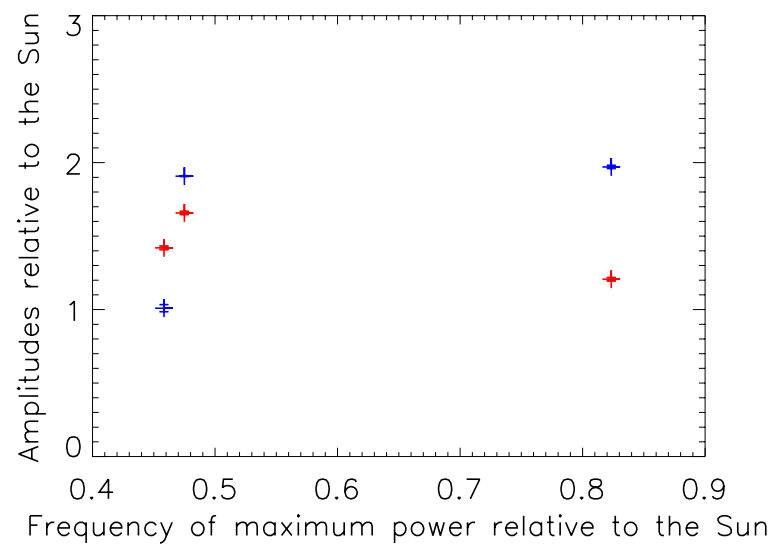

Figure 3. Measured amplitudes of the components attributed to granulation (red) and faculae (blue) as a function of the frequency of maximum power relative to the Sun. No general trend can be seen.

In order to test that the measured significances of the faculae components were not affected by using solar values for the exponents in Equation (2) we also calculated the logarithmic likelihood ratios allowing the exponents to vary around the solar values. This did change the returned logarithmic likelihood ratios, which are also shown in Table 5, but not the conclusion that the significance of the component attributed to faculae is one in all three stars. In other words it is clear that the conclusion that the component attributed to faculae is significantly present in all three stars is valid whether the exponents are fixed to solar values or allowed to change freely.

When the exponents were fixed to solar values, the exponent of the granulation component was the same in both models, but when the exponents were allowed to change freely this was not the case-here the values of the granulation exponent were generally lower in the model with no facular component. Generally, for all the tests we did, the exponents were returned with values between 3 and 5 for the component attributed to granulation and 6 and 8 for the component attributed to faculae. For the model without the faculae component the exponents were returned with values between 1.7 and 4 .

The returned logarithmic likelihood ratios also agrees with the impression from a visual inspection-that the signature of the component attributed to faculae is strongest in KIC 6603624 and weakest in KIC 6933899. In fact, as can be seen in Figure 1, it was not possible to get a satisfactory agreement between the 

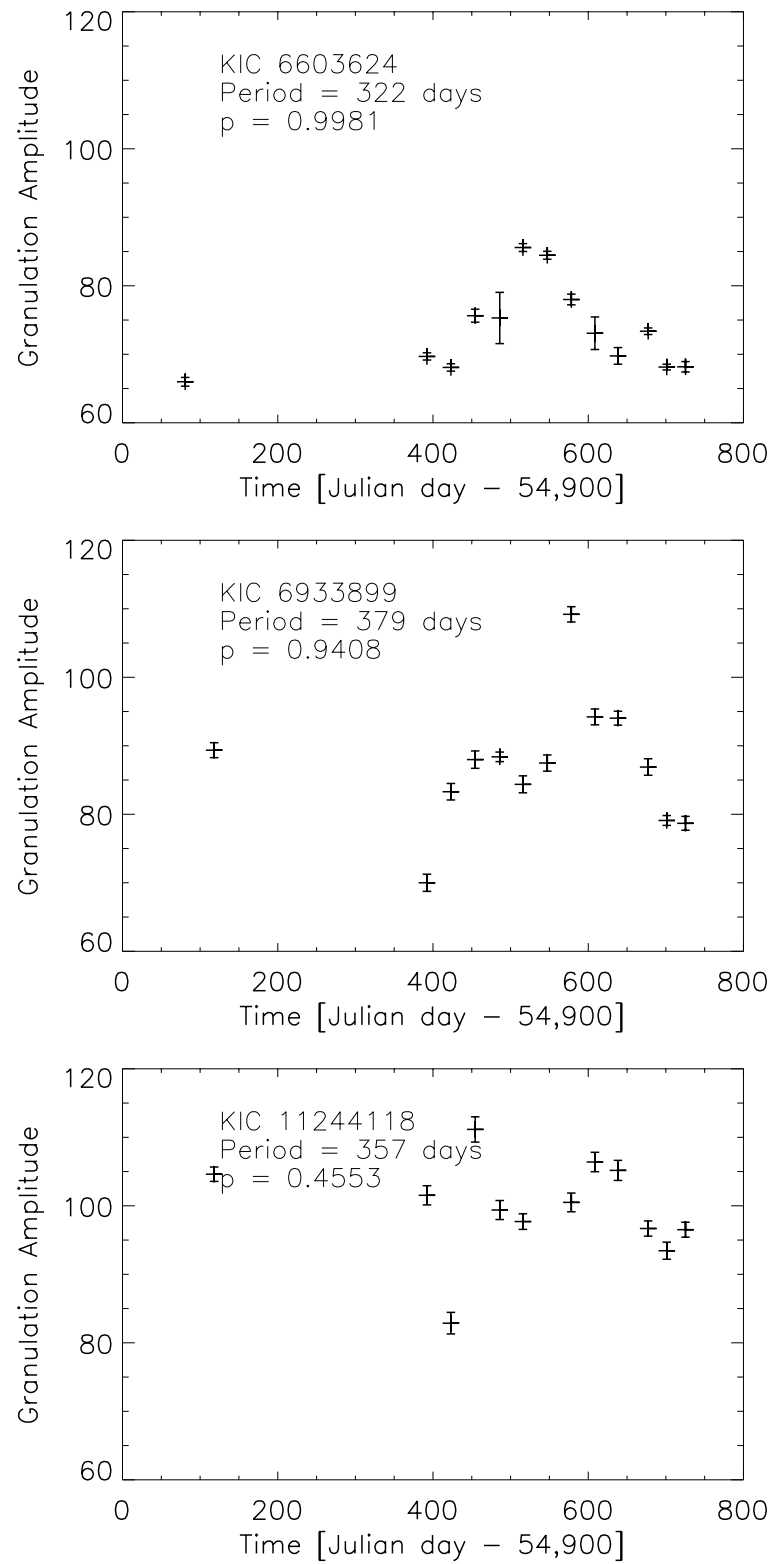

Figure 4. Temporal variability of the amplitude of the component attributed to granulation. Periodic variability with a significance level above 0.99 and a period of $322 \pm 11$ days is seen in KIC 6603624 .

model with no component attributed to faculae and the observed spectrum of KIC 6603624.

\subsection{Mean Values of the Measured Parameters}

The mean values of the measured parameters and their uncertainties were calculated from the values measured in the 13 spectra of the individual months (see Table 6).

It is well known that there exists a relation between the frequency of maximum power and the large frequency separation, which again depends on the mean stellar density (Stello et al. 2009). This agrees nicely with the fact that KIC 6603624 with a density almost twice as large as the two other stars is also the stars with the largest frequency of maximum power (see Table 6). The mean density of KIC 6603624 is still lower than the mean density of the Sun and so is the frequency of maximum power.

Even though three stars are far from enough for calculating scaling relations we have tried to compare both the timescales
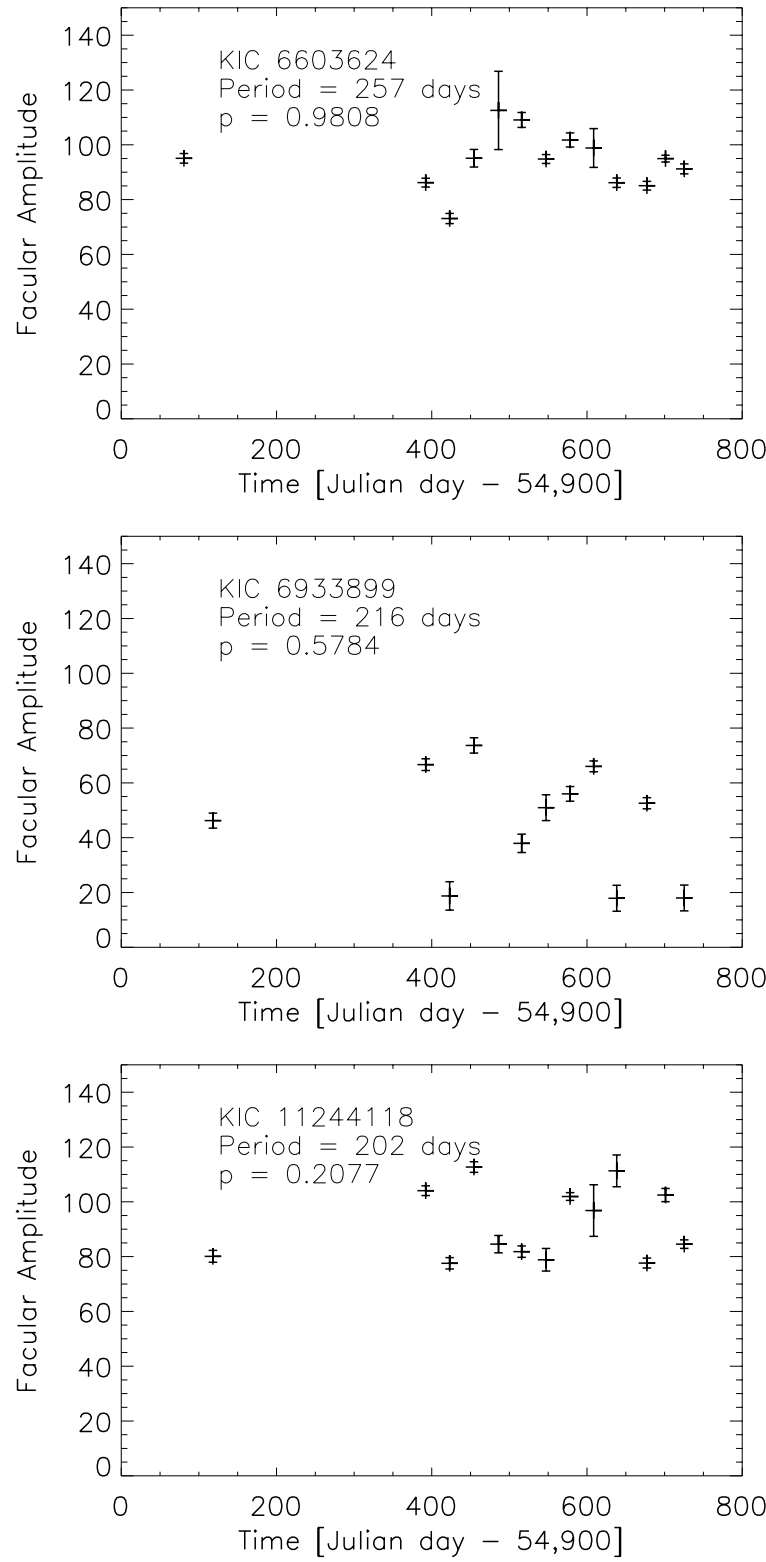

Figure 5. Temporal variability of the amplitude of the component attributed to faculae. Marginal significant periodic variability with a significance level above 0.95 and a period of $257 \pm 11$ days is seen in KIC 6603624 .

and amplitudes of the various components to the frequency of maximum power in Figures 2 and 3. It is seen that the timescales of both the components scale inversely with the frequency of maximum power (Figure 2). We do not see any clear relation between the amplitudes of the components and the frequency of maximum power (Figure 3 ). This contradicts the predictions by Chaplin et al. (2011a) that the amplitudes should scale inversely with the square of the frequency of maximum power. The reason for this is most likely that we only analyze three stars in this study.

\subsection{Temporal Variability}

We have measured the temporal variability of the background and oscillation envelope parameters in the 13 spectra of the individual months for each star. The temporal variability of the parameters of the components attributed to granulation and faculae is shown in Figures 4-7. The white noise component is shown for comparison in Figure 8. 
Table 6

Mean Parameters of the Three Stars and the Sun

\begin{tabular}{lccrcc}
\hline \hline & KIC 6603624 & KIC 6933899 & KIC 1124418 & Sun & \\
\hline$\sigma_{\text {gran }}$ & $62.8 \pm 1.5(1)$ & $74.5 \pm 2.3(1)$ & $87.4 \pm 2.9(1)$ & $62.4 \pm 0.6$ & $\mathrm{ppm}$ \\
$\tau_{\text {gran }}$ & $280.8 \pm 5.9(4)$ & $474.3 \pm 15.4(10)$ & $448.0 \pm 10.7(3)$ & $214.3 \pm 2.9$ & $\mathrm{~s}$ \\
$\sigma_{\text {fac }}$ & $76.5 \pm 2.4(1)$ & $28.7 \pm 7.5(1)$ & $74.7 \pm 3.1(1)$ & $50.1 \pm 0.1$ & $\mathrm{ppm}$ \\
$\tau_{\text {fac }}$ & $66.1 \pm 1.3(3)$ & $180.1 \pm 2.4(7)$ & $153.1 \pm 1.6(7)$ & $65.8 \pm 0.3$ & $\mathrm{~s}$ \\
$H_{\text {osc }}$ & $1.4 \pm 0.1(0.03)$ & $2.1 \pm 0.2(0.09)$ & $4.5 \pm 0.3(0.1)$ & $6.2 \pm 2.5$ & $\mathrm{ppm}^{2} \mu \mathrm{Hz}^{-1}$ \\
$\nu_{\text {max }}$ & $2477 \pm 12(8)$ & $1368 \pm 10(5)$ & $1426 \pm 14(5)$ & $3104 \pm 36$ & $\mu \mathrm{Hz}$ \\
$w$ & $246.5 \pm 0.9(10)$ & $138.9 \pm 2.0(8)$ & $233.5 \pm 2.9(7)$ & $316 \pm 36$ & $\mu \mathrm{Hz}^{-1}$ \\
Noise & $0.4 \pm 0.1(0.1)$ & $4.1 \pm 0.1(0.1)$ & $2.8 \pm 0.4(0.1)$ & & $\mathrm{ppm}^{2} \mu \mathrm{Hz}^{-1}$ \\
\hline
\end{tabular}

Notes. Definitions of the different parameters are given by Equation (2). The numbers in brackets are the uncertainties returned by the MultiNest algorithm.
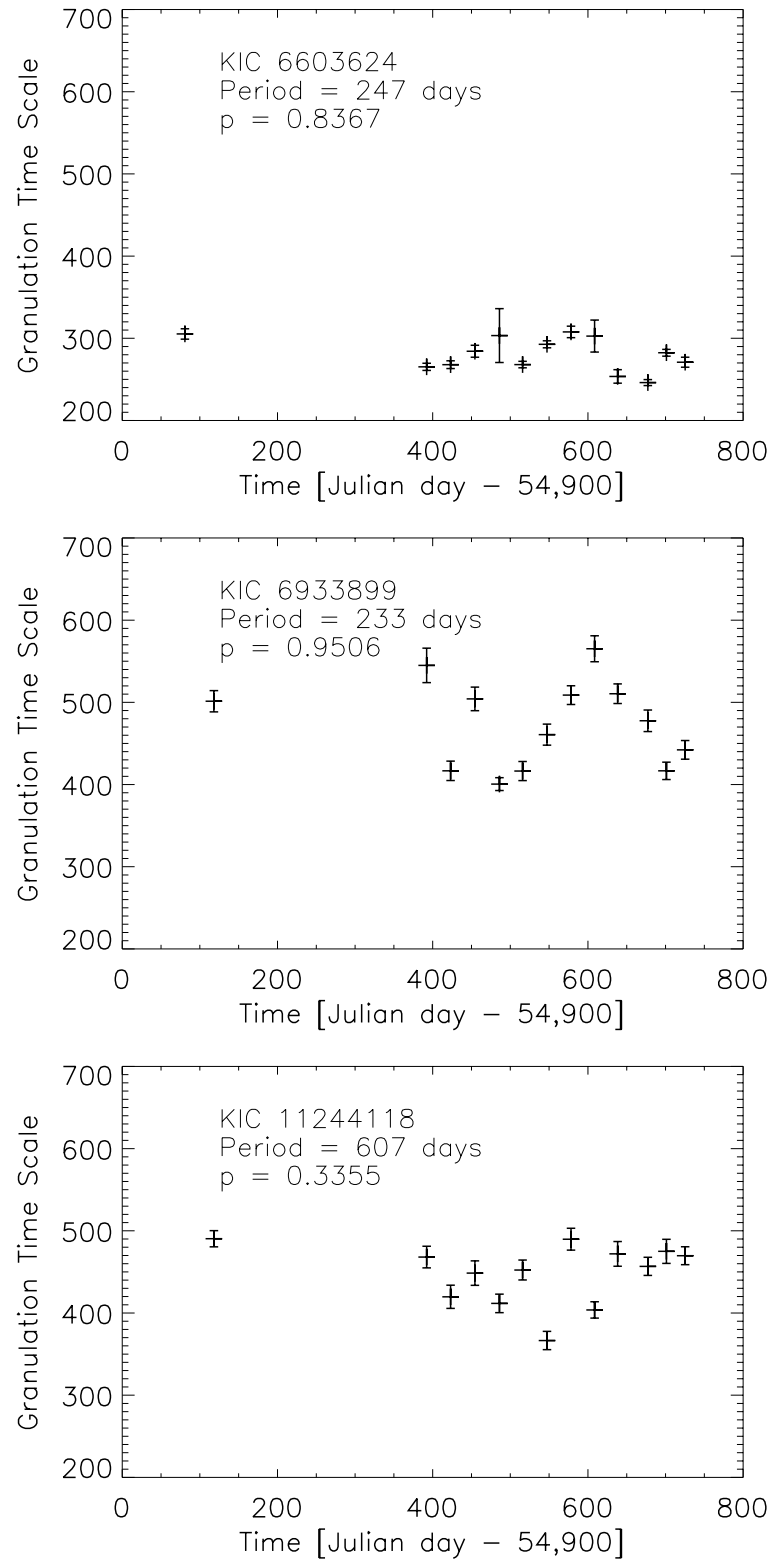

Figure 6. Temporal variability of the granulation timescale. Marginal significant periodic variability with a significance level above 0.95 and a period of $233 \pm 13$ days is seen in KIC 6933899 .

In order to test if any of the parameters show periodic variability we found the highest peak in the periodogram of the parameters as a function of time and performed a simple test of the significance of this peak. The test was performed by
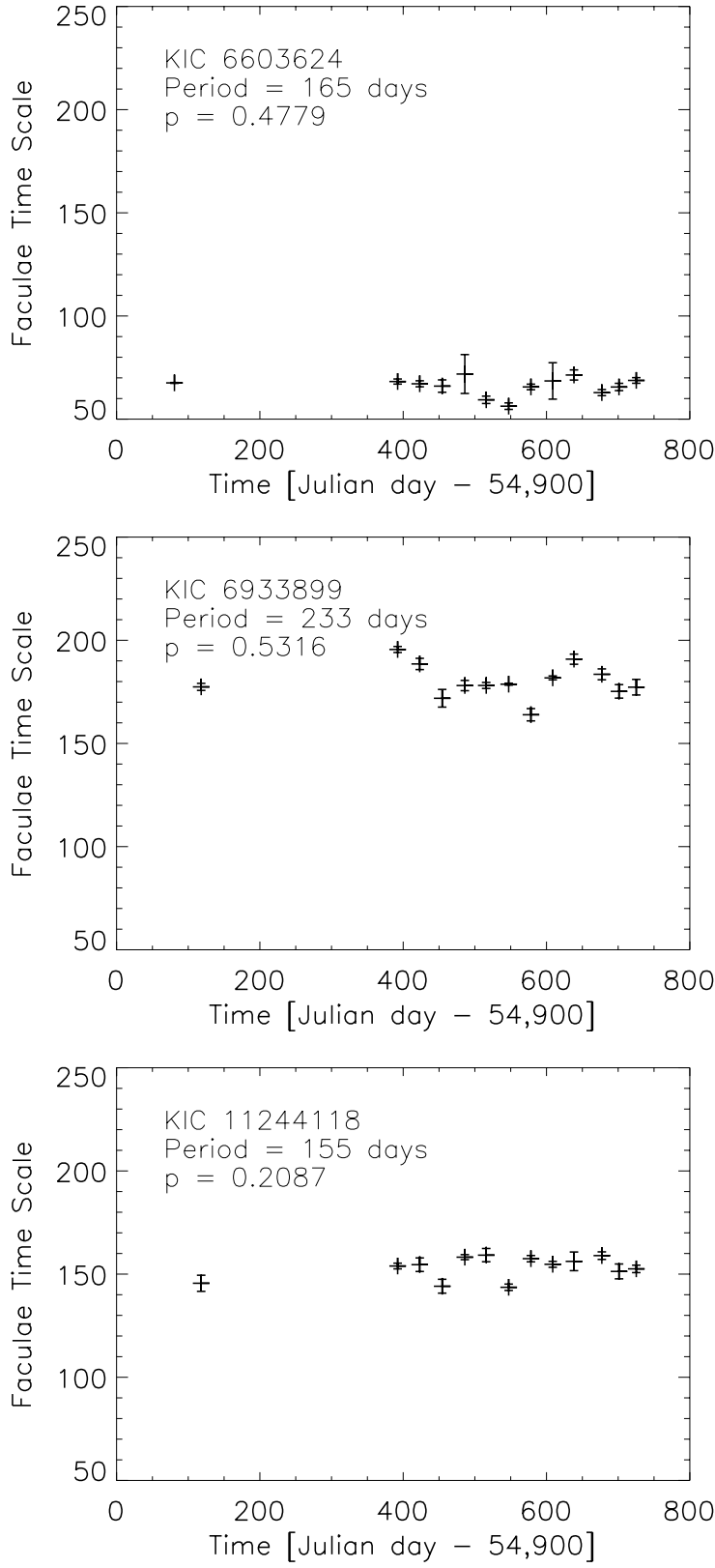

Figure 7. Temporal variability of the facular timescale.

measuring the amplitude of the highest peak in one million artificially generated periodograms. These periodograms were generated by taking the uncertainties ascribed to each measurement at each time step (calculated as the diagonal elements of the 

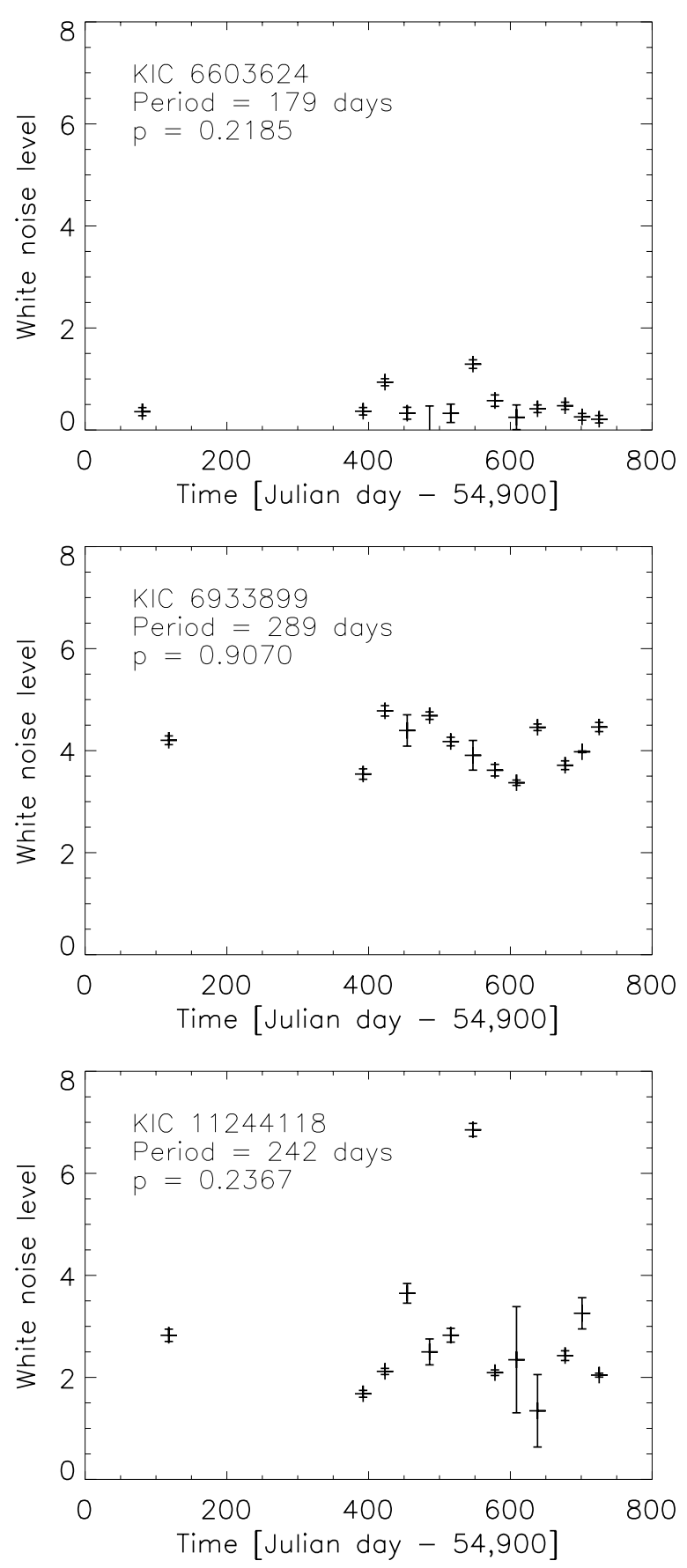

Figure 8. Temporal variability of the white noise level.

inverse of the Hessian matrix) and multiply them with a normally distributed random number with a mean of zero and a standard deviation of one. The significance was then calculated as the fraction of cases where the highest peak in these one million artificial generated periodograms had an amplitude lower than the amplitude of the highest peak in the observed periodogram.

Here we adopt the definition that a period with a significance level above 0.99 is significant, whereas a period with a significance level above 0.95 is marginal significant. This leads to the conclusion that we see a significant period of $322 \pm 11$ days (error bars calculated assuming normal distributed noise on the measured amplitudes) in the amplitude of the component attributed to granulation in KIC 6603624. Marginal significant periods are found in the amplitude of the component attributed
Table 7

Correlation between the Faculae and Granulation Parameters (KIC 6603624/KIC 6933899/KIC 11244118)

\begin{tabular}{lccr}
\hline \hline & $\sigma_{\text {fac }}$ & $\tau_{\text {gran }}$ & \multicolumn{1}{c}{$\tau_{\text {fac }}$} \\
\hline$\sigma_{\text {gran }}$ & $0.56 / 0.21 / 0.09$ & $0.15 / 0.26 /-0.47$ & $-0.72 /-0.56 /-0.61$ \\
\hline$\sigma_{\text {fac }}$ & & $0.59 / 0.74 / 0.36$ & $-0.09 / 0.01 /-0.08$ \\
\hline$\tau_{\text {gran }}$ & & & $0.06 / 0.18 / 0.18$ \\
\hline
\end{tabular}

to faculae in KIC 6603624 (257 \pm 11 days) and in the timescale of the component attributed to granulation in KIC 6933899 ( $233 \pm 13$ days). The significant levels of the highest peaks in the periodograms of the temporal variability of the granulation and faculae parameters for the three stars are given in Figures 4-7. No significant or marginal significant periods were found in the parameters of the envelope of the $p$-mode oscillations.

The periods of the periodic variability seen in the components attributed to granulation and faculae in KIC 6603624 and KIC 6933899 are between 200 and 300 days. This could be equivalent to the quasi-annual modulation of the amplitude of the component attributed to granulation in the frequency spectrum of intensity observations of the Sun with the Variability of solar IRradiance and Gravity Oscillations (Fröhlich et al. 1995) instrument on the Solar and Heliospheric Observatory (SOHO; Karoff 2012). Unfortunately, it has been impossible to study such a modulation in velocity observations with Global Oscillations at Low Frequency (Gabriel et al. 1995) instrument also on $\mathrm{SOHO}$ due to a variation of the observation height in the solar atmosphere induced by the orbital period of the satellite (Lefebvre et al. 2008).

Even though none of the identified periods are identical we did calculate the linear Pearson correlation coefficients between the granulation and faculae parameters (see Table 7). The numerical values of these correlation coefficients were generally very low. The largest one being between the amplitude of the component attributed to faculae and the timescale of the component attributed to granulation in KIC 6922899 with a value of 0.74 . The low levels of these correlation coefficients verify that there are no correlations between the different parameters and thus that the identified periods are intrinsic to the amplitude of the component attributed to granulation in KIC 6603624, the amplitude of the component attributed to faculae in KIC 6603624, and the timescale attributed to granulation in KIC 6933899, respectively.

\subsection{Comparison of Different Analysis Methods}

It was shown by Mathur et al. (2011) that different analysis methods with different free parameters, models, data, and number of components can provide different values of the estimated parameters, but that, for a given method, the results and the trends are consistent. Though we do not want to redo this analysis here it is still interesting to investigate if the component attributed to faculae can also be found using different analysis methods.

We therefore redid the analysis of the frequency spectra of the three stars using the CAN and A2Z methods from Mathur et al. (2011; see Mathur et al. 2010; Kallinger et al. 2010 for a detailed description of the methods) and a method that used the same model as described here, but no binning of the spectra and thus assuming that the differences between the observed frequency spectra and the model were given by a $\chi^{2}$ distribution with 2 degrees of freedom. 
The result of the comparison was that the component attributed to faculae was significantly present in the observed frequency spectra with a significance close to 1 in all three stars in all the tests where convergence to physically meaningful parameters could be reached.

The comparison also showed that the uncertainties on the mean parameter values are realistic-i.e., they are comparable in size to the difference between the different analysis methods and the uncertainties returned by the MultiNest algorithm used in the CAN methods (which have been shown in brackets for comparison in Table 6). The error bars on the temporal results calculated from the diagonal elements of the inverse of the Hessian matrix are on the other hand generally too low (as also noted by Mathur et al. 2011).

\section{CONCLUSIONS}

The analysis of the observed frequency spectra of the 3 Sunlike stars KIC 6603624, KIC 6933899, and KIC 11244118 from 13 months of high-precision, high-cadence photometric observations from the Kepler spacecraft has revealed signatures of what is likely granulation, faculae, and $p$-mode oscillations in all 3 stars.

It is seen that the characteristic frequencies (or equivalent, timescales) of the components attributed to both granulation and faculae scale inversely with the frequency of maximum power and that the frequency of maximum power scale with the mean density of the stars as expected as the atmospheric acoustic cutoff frequency scales with the large frequency separation (Stello et al. 2009).

The analysis of the temporal variability of the measured parameters revealed periodic variability in the amplitude of the component attributed to granulation in KIC 6603624 with a significance level above 0.99 and a period of $322 \pm 11$ days. Marginal significant periodic variability with a significance level above 0.95 was found in the amplitude of the component attributed to faculae in KIC 6603624 with a period of $257 \pm$ 11 days and in the timescale of the component attributed to granulation in KIC 6933899 with a period of $233 \pm 13$ days.

The temporal variability in KIC 6603624 and KIC 6933899 could have an origin similar to the periodicities of around 1-2 yr seen in a number of indices related to solar activity, including: sunspot number and neutrino flux (Sakurai 1979), Galactic cosmic-ray intensities (Valdés-Galicia et al. 1996), flare occurrence (Antalova 1994), solar wind velocities (Richardson et al. 1994), aa geomagnetic indices (Mursula et al. 2003), coronal hole area and radio emission (Valdés-Galicia \& Velasco 2008), the rotation of the Sun near the base of its convective zone (Howe et al. 2000), and lately in the residuals of the $p$-mode frequency shifts (Fletcher et al. 2010; Broomhall et al. 2012; Simoniello et al. 2013).

The periods of the periodic variability in KIC 6603624 (and KIC 6933899) are between two hundred and three hundred days, which is somewhat lower than the 1-2 yr periodicities observed in the indices related to solar activity. This agrees with the lower densities of these stars compared to the Sun, but it is not clear if it agrees with the period of any possible dynamo in these stars.

We thank the referee for thoughtful comments, which significantly improved the paper. Funding for this Discovery mission is provided by NASAs Science Mission Directorate. The authors wish to thank the entire Kepler team, without whom these results would not be possible. C.K. acknowledged support from the Carlsberg foundation. T.L.C. acknowledges financial support from project PTDC/CTE-AST/098754/2008 funded by FCT/MCTES, Portugal. T.L.C. also acknowledges the support of the UK Science and Technology Facilities Council (STFC). T.K. is supported by the FWO-Flanders under project O6260 - G.0728.11. Funding for the Stellar Astrophysics Centre is provided by The Danish National Research Foundation (grant agreement No.: DNRF106). The research is supported by the ASTERISK project (ASTERoseismic Investigations with SONG and Kepler) funded by the European Research Council (grant agreement No.: 267864).

\section{REFERENCES}

Aigrain, S., Favata, F., \& Gilmore, G. 2004, in Stellar Structure and Habitable Planet Finding, ed. F. Favata, S. Aigrain, \& A. Wilson (ESA SP-538; Noordwijk: ESA), 215

Andersen, B., Leifsen, T., Appourchaux, T., et al. 1998, Structure and Dynamics of the Interior of the Sun and Sun-like Stars (ESA SP-418; Noordwijk: ESA), 418,83

Anklin, M., Frohlich, C., Wehrli, C., \& Finsterle, W. 1998, Structure and Dynamics of the Interior of the Sun and Sun-like Stars (ESA SP-418; Noordwijk: ESA), 418, 91

Antalova, A. 1994, AdSpR, 14, 721

Appourchaux, T. 2003, A\&A, 412, 903

Appourchaux, T. 2004, A\&A, 428, 1039

Appourchaux, T., Chaplin, W. J., García, R. A., et al. 2012, A\&A, 543, A54

Belkacem, K., Goupil, M. J., Dupret, M. A., et al. 2011, A\&A, 530, A142

Broomhall, A.-M., Chaplin, W. J., Elsworth, Y., \& Simoniello, R. 2012, MNRAS, 420, 1405

Brown, T. M., Gilliland, R. L., Noyes, R. W., \& Ramsey, L. W. 1991, ApJ, 368,599

Chaplin, W. J., Appourchaux, T., Elsworth, Y., et al. 2010, ApJL, 713, L169

Chaplin, W. J., Kjeldsen, H., Bedding, T. R., et al. 2011a, ApJ, 732, 54

Chaplin, W. J., Kjeldsen, H., Christensen-Dalsgaard, J., et al. 2011b, Sci, 332,213

Del Moro, D. 2004, A\&A, 428, 1007

Fletcher, S. T., Broomhall, A.-M., Salabert, D., et al. 2010, ApJL, 718, L19

Fröhlich, C., Romero, J., Roth, H., et al. 1995, SoPh, 162, 101

Gabriel, M. 1994, A\&A, 287, 685

Gabriel, A. H., Grec, G., Charra, J., et al. 1995, SoPh, 162, 61

García, R. A., Hekker, S., Stello, D., et al. 2011, MNRAS, 414, L6

García, R. A., Pallé, P. L., Turck-Chièze, S., et al. 1998, ApJL, 504, L51

Gilliland, R. L., Jenkins, J. M., Borucki, W. J., et al. 2010, ApJL, 713, L160

Goldreich, P., Murray, N., \& Kumar, P. 1994, ApJ, 424, 466

Harvey, J. 1985, Future Missions in Solar, Heliospheric and Space Plasma Physicenvelopes (ESA SP-235; Noordwijk: ESA), 235, 199

Harvey, J. W., Duvall, T. L., Jr., Jefferies, S. M., \& Pomerantz, M. A. 1993, in ASP Conf. Ser. 42, GONG 1992, Seismic Investigation of the Sun and Stars, ed. T. M. Brown (San Francisco, CA: ASP), 111

Houdek, G., Balmforth, N. J., Christensen-Dalsgaard, J., \& Gough, D. O. 1999, A\&A, 351, 582

Howe, R., Christensen-Dalsgaard, J., Hill, F., et al. 2000, Sci, 287, 2456

Jenkins, J. M., Caldwell, D. A., Chandrasekaran, H., et al. 2010, ApJL, 713, L87

Jiménez, A., Jiménez-Reyes, S. J., \& García, R. A. 2005, ApJ, 623, 1215

Kjeldsen, H., \& Bedding, T. R. 1995, A\&A, 293, 87

Kjeldsen, H., \& Bedding, T. R. 2011, A\&A, 529, L8

Kallinger, T., Mosser, B., Hekker, S., et al. 2010, A\&A, 522, A1

Karoff, C. 2007, MNRAS, 381, 1001

Karoff, C. 2008, PhD dissertation, Aarhus University

Karoff, C. 2009, in ASP Conf. Ser. 416, Solar-Stellar Dynamos as Revealed by Helio- and Asteroseismology: GONG 2008/SOHO 21, ed. M. Dikpati, T. Arentoft, I. G. Hernández, C. Lindsey, \& F. Hill (San Francisco, CA: ASP), 233

Karoff, C. 2012, MNRAS, 421, 3170

Karoff, C., \& Kjeldsen, H. 2008, ApJL, 678, L73

Koch, D. G., Borucki, W. J., Basri, G., et al. 2010, ApJL, 713, L79

Lanza, A. F., Rodonò, M., \& Pagano, I. 2004, A\&A, 425, 707

Lefebvre, S., García, R. A., Jiménez-Reyes, S. J., Turck-Chièze, S., \& Mathur, S. 2008, A\&A, 490, 1143

Lomb, N. R. 1976, Ap\&SS, 39, 447

Mathur, S., García, R. A., Régulo, C., et al. 2010, A\&A, 511, A46

Mathur, S., Hekker, S., Trampedach, R., et al. 2011, ApJ, 741, 119

Mathur, S., Metcalfe, T. S., Woitaszek, M., et al. 2012, ApJ, 749, 152 
Michel, E., Baglin, A., Auvergne, M., et al. 2008, Sci, 322, 558

Michel, E., Samadi, R., Baudin, F., et al. 2009, A\&A, 495, 979

Mursula, K., Zieger, B., \& Vilppola, J. H. 2003, SoPh, 212, 201

Nordlund, A., Spruit, H. C., Ludwig, H.-G., \& Trampedach, R. 1997, A\&A, 328,229

Press, W. H., Teukolsky, S. A., Vetterling, W. T., \& Flannery, B. P. 1992, Numerical Recipes in C (2nd ed.; Cambridge: Cambridge Univ. Press)

Richardson, J. D., Paularena, K. I., Belcher, J. W., \& Lazarus, A. J. 1994, GeoRL, 21, 1559

Roudier, T., Malherbe, J. M., Vigneau, J., \& Pfeiffer, B. 1998, A\&A, 330, 1136
Sakurai, K. 1979, Natur, 278, 146

Schwarzschild, M. 1975, ApJ, 195, 137

Simoniello, R., Jain, K., Tripathy, S. C., et al. 2013, ApJ, 765, 100

Stello, D., Chaplin, W. J., Basu, S., Elsworth, Y., \& Bedding, T. R. 2009, MNRAS, 400, L80

Valdés-Galicia, J. F., Pérez-Enríquez, R., \& Otaola, J. A. 1996, SoPh, 167, 409

Valdés-Galicia, J. F., \& Velasco, V. M. 2008, AdSpR, 41, 297

Vázquez Ramió, H., Roca Cortés, T., \& Régulo, C. 2002, in Solar Variability: From Core to Outer Frontiers, ed. A. Wilson (ESA SP-506; Noordwijk: ESA), 897

Verner, G. A., Elsworth, Y., Chaplin, W. J., et al. 2011, MNRAS, 415, 3539 